\title{
CORRESPONDENCE Sunny walking counts more
}

Rui Zhang ${ }^{1}$, Hong Chen ${ }^{1}$, Yi-xiao Liu' ${ }^{1}$, Wen-hui Zhang ${ }^{1}$, Qin Lu' ${ }^{1}$, Hiromichi Yamanishi ${ }^{2}$, Chiaki Yamanishi ${ }^{2}$, Kyosuke Yamanishi ${ }^{2}$, Yu-lan Qiu' ${ }^{3}$, Xiao-fei Ye ${ }^{3}$, Zi-rui Huang ${ }^{1}$, Bo-yuan Zhang ${ }^{1}$, Yi-fei Chen ${ }^{1}$, Yan-qian Zheng ${ }^{1}$, Yong-fang Zhang ${ }^{1}$, Zi-zhen Guo ${ }^{1}$, Dong Dong ${ }^{1}$, Tie-xin Liu' ${ }^{1}$, Yi-qing Dai ${ }^{1}$, Mei-han Xu' ${ }^{1},{ }^{1}$ Y Hao ${ }^{1}$, Sheng-zhou Li ${ }^{1}$, Fei-yang Cai ${ }^{1}$, Rui-qi Wang ${ }^{1}$, Xin-yi Guo ${ }^{1}$, De-hao Zhu' Hai-yang Zhang ${ }^{1}$, Zhi-tong Zeng ${ }^{1}$ and Hideaki Higashino ${ }^{2}$

Acta Pharmacologica Sinica (2019) 40:1256-1257; https://doi.org/10.1038/s41401-019-0241-9

\section{Dear Editor,}

In our previous article entitled "Low-intensity walking as mild medication for pressure control in prehypertensive and hypertensive subjects: how far shall we wander?" published in this journal [1], we reported mild but significant reductions in both blood pressure $(\mathrm{BP})$ and heart rate $(\mathrm{HR})$ after low-intensity walking at a speed of $3 \mathrm{~km} / \mathrm{h}$ (2.5 metabolic equivalents of task, METs), accompanied by a transient elevation in urine $\beta$-endorphin. In a recent study observing environmental influence on low-intensity walking, we found unexpectedly interesting results that we would like to share with the readers of the journal.

In the aforementioned study, walking was usually performed in the open air during the day but occasionally indoors when it was raining or either too cold or too hot outside. We further designed a study of the difference between low-intensity walking with sunshine (sunny walking) and without sunshine (sunless walking) for $1 \mathrm{~h}$ in normotensive subjects aged 19-74 years. Sunny walking was performed on sunny days in open venues where the participants walked in the sun (sunbathed). Sunless walking was performed indoors with only indoor lamplight or outdoors with minimal sunlight on overcast days.

It was interesting to find that sunny walking and sunless walking resulted in similar reductions in resting HR but discrete effects on BP. As shown in Table 1, sunny walking caused a brief but significant decrease in both resting $\mathrm{BP}$ and $\mathrm{HR}$, as we observed previously $[1,2]$. Sunless walking, however, produced no clinically meaningful reduction in BP in addition to the reduced HR. Sunny walking also resulted in elevated urine $\beta$-endorphin output during walking, whereas sunless walking did not. The lack of $\beta$-endorphin change during sunless walking was contrary to our previous proposal that low-intensity walking per se elevated $\beta$-endorphin, which might partly count for the walking-related reduction in BP. $\beta$-Endorphin was tested with a previously used enzyme-linked immunosorbent assay (Wuhan Elabscience Biotechnology Co., Ltd, China) [1, 2]. These findings suggest that low-intensity walking reduced HR independently of $\beta$-endorphin. Different mechanisms should be involved in the cardiovascular effects of walking in different environments. Additionally, sunny walking has a greater effect.

Sunny walking elevated the surface temperature of the extremities (palm) but not that of the forehead (Table 1). Sunless walking, in contrast, decreased the temperature of both the palm and forehead. The warming of the extremities by the sun during walking could promote circulation and vasodilation of the skin vasculature that may contribute to $B P$ reduction in addition to $\beta$ endorphin. The surface temperature reduction during walking with no sunlight exposure might be caused by walking-enhanced

\begin{tabular}{|c|c|c|c|c|}
\hline & $\begin{array}{l}\text { Sunny } \\
\text { walking }\end{array}$ & $\Delta$ & $\begin{array}{l}\text { Sunless } \\
\text { walking }\end{array}$ & $\Delta$ \\
\hline$n$ & 35 & & 35 & \\
\hline Age, year & $22.8 \pm 9.2$ & & $25.2 \pm 13.2$ & \\
\hline $\begin{array}{l}\text { Body weight, } \\
\mathrm{kg} / \mathrm{m}^{2}\end{array}$ & $21.5 \pm 2.2$ & & $21.3 \pm 2.1$ & \\
\hline \multicolumn{5}{|c|}{ Mean arterial pressure $(\mathrm{mmHg})$} \\
\hline Prewalk & $87 \pm 8$ & & $86 \pm 8$ & \\
\hline Postwalk & $84 \pm 7^{* *}$ & $-3.1 \pm 4.9$ & $86 \pm 7$ & $-0.4 \pm 5.3$ \\
\hline \multicolumn{5}{|c|}{ Heart rate (beats/min) } \\
\hline Prewalk & $83 \pm 13$ & & $78 \pm 11$ & \\
\hline Postwalk & $78 \pm 13^{* *}$ & $-4.9 \pm 7.5$ & $74 \pm 12^{* *}$ & $-5.2 \pm 5.4$ \\
\hline \multicolumn{5}{|c|}{ Forehead surface temperature $\left({ }^{\circ} \mathrm{C}\right)$} \\
\hline Prewalk & $36.6 \pm 0.4$ & & $36.4 \pm 0.8$ & \\
\hline Postwalk & $36.6 \pm 0.3$ & $0.00 \pm 0.36$ & $36.2 \pm 0.9^{* *}$ & $-0.25 \pm 0.48$ \\
\hline \multicolumn{5}{|c|}{ Palm surface temperature $\left({ }^{\circ} \mathrm{C}\right)$} \\
\hline Prewalk & $35.8 \pm 1.1$ & & $36.0 \pm 1.1$ & \\
\hline Postwalk & $36.3 \pm 0.8^{*}$ & $0.56 \pm 0.99$ & $35.0 \pm 1.7^{* *}$ & $-1.05 \pm 1.64$ \\
\hline \multicolumn{5}{|c|}{ Urine $\beta$-endorphin output (pg/h) } \\
\hline Prewalk & $4458 \pm 3572$ & & $6402 \pm 4934$ & \\
\hline Postwalk & $7280 \pm 6623^{*}$ & $2822 \pm 6708$ & $5731 \pm 4255$ & $-670 \pm 4589$ \\
\hline \multicolumn{5}{|c|}{ Log-transformed urine $\beta$-endorphin, log (pg/h) } \\
\hline Prewalk & $3.55 \pm 0.28$ & & $3.67 \pm 0.37$ & \\
\hline Postwalk & $3.74 \pm 0.31 * *$ & $0.19 \pm 0.37$ & $3.63 \pm 0.38$ & $-0.04 \pm 0.43$ \\
\hline \multicolumn{5}{|c|}{ Urine albumin output $(\mu \mathrm{g} / \mathrm{h})$} \\
\hline Prewalk & $214 \pm 210$ & & $326 \pm 286$ & \\
\hline Postwalk & $275 \pm 300$ & $60 \pm 318$ & $221 \pm 185$ & $-105 \pm 279$ \\
\hline \multicolumn{5}{|c|}{ Log-transformed urine albumin, log $(\mu \mathrm{g} / \mathrm{h})$} \\
\hline Prewalk & $2.20 \pm 0.33$ & & $2.31 \pm 0.47$ & \\
\hline Postwalk & $2.20 \pm 0.50$ & $0.00 \pm 0.53$ & $2.24 \pm 0.31$ & $-0.09 \pm 0.47$ \\
\hline
\end{tabular}

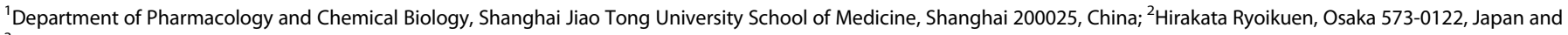
${ }^{3}$ Department of Epidemiology and Biostatistics, Shanghai Jiao Tong University School of Public Health, Shanghai 200025, China Correspondence: Hong Chen (hchen100@shsmu.edu.cn) or Hideaki Higashino (hhigasino3@osaka.zaq.jp)

These authors contributed equally: Rui Zhang, Hong Chen
}

Received: 20 February 2019 Accepted: 25 April 2019

Published online: 27 June 2019 
circulation that promoted the dissipation of body heat without much heat-related cutaneous vasodilation.

Sunlight includes a broad spectrum of wavelengths, including ultraviolet (UV), far-infrared and infrared rays, which have been shown to affect cardiovascular function in different studies [35]. It was reported that whole body UV radiation was associated with the cutaneous release of enzyme-independent nitric oxide (NO) and a greater decrease in BP than was produced by skin heating $[3,4]$. During our sunny walking, a limited area of body skin (i.e., the head and hands) was exposed to sunlight. It remains to be determined which contributes more to lowering BP during sunny walking, UV radiation-related NO, far-infrared/ infrared radiation, the heating of the body, or other vasodilating factors.

Collectively, our data suggest that sunny walking lowers BP and HR by multiple mechanisms. Mild exercise in the open air with sunlight exposure may provide additional benefits to the cardiovascular system in hypertensive individuals who perform little outdoor physical activity.

\section{ACKNOWLEDGEMENTS}

This work was supported by grants from the National Natural Science Foundation of China to Hong Chen (No. 30971154, 31171099) and Wen-hui Zhang (No. 81373468). We are grateful to Dr. Hai Su, Wei-li Shen and Yoshinori Aragane for engaging in active discussions.

\section{AUTHOR CONTRIBUTIONS}

All authors contributed to performing the research by participating in the walking process and making records and/or by helping to develop the research; HC, RZ, QL and $\mathrm{Y}-\mathrm{xL}$ collected urine samples, performed ELISA tests, and analyzed the data; Hong Chen and Hideaki Higashino designed the research and wrote and edited the manuscript; and all authors reviewed and approved the manuscript.

\section{ADDITIONAL INFORMATION}

Competing interests: The authors declare no competing interests.

\section{REFERENCES}

1. Lu Q, Wang S, Liu YX, Chen H, Zhang R, Zhang WH, et al. Low-intensity walking as mild medication for pressure control in prehypertensive and hypertensive subjects: how far shall we wander? Acta Pharmacol Sin. 2019; 40: in press. https://doi. org/10.1038/s41401-018-0202-8.

2. Wang SM, Chen YC, Lu Q, Wang J, Zhong MF, Chen H, et al. Low-speed walking for three kilometers was effective to lower short-term blood pressure and heart rate with increased $\beta$-endorphin release in normotensive and hypertensive subjects. Chin J Hypertens. 2018;26:953-61.

3. Oplander C, Volkmar CM, Paunel-Gorgulu A, van Faassen EE, Heiss C, Kelm M, et al. Whole body UVA irradiation lowers systemic blood pressure by release of nitric oxide from intracutaneous photolabile nitric oxide derivates. Circ Res. 2009;105:1031-40.

4. Liu D, Fernandez BO, Hamilton A, Lang NN, Gallagher JM, Newby DE, et al. UVA irradiation of human skin vasodilates arterial vasculature and lowers blood pressure independently of nitric oxide synthase. J Invest Dermatol. 2014;134:1839-46.

5. Aragane $\mathrm{Y}$, Higashino $\mathrm{H}$. The "Enseki" sandbath: A novel, safe and effective farinfrared bathing procedure for health. Photodermatol Photoimmunol Photomed. 2019;35:31-9. 